Militaires et sécurité intérieure.

\title{
Guantanamo. Sortir du silence, refuser l'impunité
}

Entretien avec Maître William Bourdon

\section{Didier Bigo et Christian Olsson}

\section{(2) OpenEdition \\ Journals}

Édition électronique

URL : http://journals.openedition.org/conflits/1626

DOI : $10.4000 /$ conflits. 1626

ISSN : $1777-5345$

Éditeur :

CCLS - Centre d'études sur les conflits lilberté et sécurité, L'Harmattan

Édition imprimée

Date de publication : 1 décembre 2004

Pagination : 71-81

ISBN : 2-7475-7598-X

ISSN : 1157-996X

Référence électronique

Didier Bigo et Christian Olsson, "Guantanamo. Sortir du silence, refuser l'impunité », Cultures \& Conflits [En ligne], 56 | hiver 2004, mis en ligne le 07 janvier 2010, consulté le 30 mars 2021. URL : http:// journals.openedition.org/conflits/1626; DOI : https://doi.org/10.4000/conflits.1626

Ce document a été généré automatiquement le 30 mars 2021.

Creative Commons License 


\title{
Guantanamo. Sortir du silence, refuser l'impunité
}

\author{
Entretien avec Maître William Bourdon
}

Didier Bigo et Christian Olsson

1 Ce texte est né de la retranscription d'un entretien réalisé en février 2004 avec Me William Bourdon, avocat depuis 2002 avec Me Debray de Mourad Benchellali et de Nizar SASSI, deux des sept prisonniers français ayant été détenus à Camp Delta à Guantanamo Bay. Il a été réactualisé à la fin du mois de décembre 2004 par Me Bourdon lui-même. Il s'agit donc d'un véritable texte d'actualité qui illustre non seulement les enjeux juridiques, politiques et éthiques de la détention arbitraire dans le camp de Guantanamo, mais aussi ceux du sort judiciaire de quatre anciens détenus depuis leur rapatriement en France en juillet 2004.

C\&C - Pouvez-vous nous rappeler pourquoi l'obligation d'appliquer le statut de prisonnier de guerre aux prisonniers à Guantanamo Bay ne fait aucun doute malgré la position contraire du gouvernement américain ?

3 La réponse est assez simple. Les Etats-Unis sont parties aux Conventions de Genève. Pourtant, ils semblent agir au travers d'une sélection de morceaux choisis de ces conventions en n'appliquant que les dispositions qui sont les moins contraignantes pour eux. Ils en tirent prétexte pour dire qu'ils appliquent le droit international puisqu'ils laissent le CICR visiter les prisonniers et prétendent garantir que les personnes arrêtées sur le terrain des hostilités sont traitées avec humanité. Il est vrai que ce dernier point constitue un élément essentiel des quatre Conventions de Genève: le belligérant ne peut se venger sur ses prisonniers. Mais, justement, le prétexte est douteux puisque cette dernière disposition ne semble pas être appliquée. Parmi les dispositions centrales qui ne sont pas respectées, il y a celle au terme de laquelle toute personne arrêtée lors d'un conflit doit être présumée prisonnier de guerre. S'il y a un doute sur son appartenance à cette catégorie, un tribunal compétent doit statuer dans les meilleurs délais sur cette question ${ }^{1}$. En d'autres termes, seul ce tribunal peut décider si la personne interceptée est un prisonnier de guerre ou pas. Jusque-là, elle doit être considérée comme prisonnier de guerre et à ce titre, par exemple, elle ne peut pas être 
interrogée. Les quatre Conventions de Genève sont donc claires: toute personne capturée sur le champ de bataille doit être présumée prisonnier de guerre jusqu'à ce qu'une cour compétente statue autrement. Les prisonniers français ont été arrêtés au Pakistan et certains provenaient de l'Afghanistan. Ils doivent à ce titre être considérés comme prisonniers de guerre et traités en conséquence ${ }^{2}$. D'ailleurs même les prisonniers à Guantanamo qui n'ont pas été arrêtés sur le champ de bataille (certains ont été arrêtés en Gambie, d'autres en Bosnie-Herzégovine - NDLR) doivent au titre de la quatrième convention de Genève également bénéficier d'une protection juridique, même si celle-ci est moindre que pour les prisonniers de guerre. Là encore, ces dispositions ne sont absolument pas respectées. Les autorités américaines, ou plutôt une partie de l'administration américaine représentée par M. Rumsfeld, a inventé cette notion de unlawful combattant (combattant illégal) qui n'existe pas dans les Conventions de Genève. Cette notion est utilisée pour affirmer que les dispositions dont on vient de parler ne s'appliqueraient pas. Mais en réalité cette violation délibérée des Conventions de Genève tient à ce que l'objectif des américains est de pouvoir interroger indéfiniment ces gens sans la présence d'un juge ou d'un avocat. Tout cela au nom de la lutte sacrée et messianique contre le terrorisme international. Les autorités américaines essayent de justifier ce contournement du droit international en prétendant, sans aucune vérification possible, que depuis trois ans les interrogatoires sur la base de Guantanamo ont permis de casser des cellules d'Al Quaïda à travers le monde. Ces informations ne peuvent pas être vérifiées. Mais, même à supposer que cela soit vrai, le non-respect du droit international pose tout de même un problème central. En effet, il n'y a pas un crime dont la gravité est telle que cela justifie de faire reculer les dispositions fondamentales protectrices des droits de l'homme.

C\&C - Il y a eu beaucoup de débats en Grande-Bretagne sur les prisonniers à Guantanamo. Plusieurs raisons peuvent être évoquées pour expliquer cela : l'activisme des ONG britanniques, le fait qu'Amnesty International soit basé à Londres, la coalition militaire avec les Etats-Unis... Il n'en reste pas moins qu'il semble y avoir un paradoxe : les ONG britanniques ont poussé le gouvernement Blair à aller bien plus loin dans la critique des positions du gouvernement américain, gouvernement avec lequel il est pourtant fortement lié, que le gouvernement français au sein duquel il y a un quasi-silence sur la question de Guantanamo. Comment analysez-vous ce phénomène?

5 Il est vrai que les autorités britanniques sont à un niveau de protestation plus élevé que les autorités françaises. Il y a plusieurs raisons à cela. Tout d'abord, la société civile anglo-saxonne a une réactivité démocratique, pour des raisons culturelles et historiques, plus forte que la société civile française. Ensuite, dans un contexte de participation très fortement contestée à la guerre contre l'Irak, la pression des parlementaires britanniques a contraint le gouvernement Blair à faire des concessions en demandant des garanties à son allié américain sur la question des prisonniers britanniques à Guantanamo. Le rôle des parlementaires ne doit pas être sous-estimé. Nous avions demandé plusieurs fois, en vain, que les autorités françaises n'en fassent pas moins que les Britanniques sur cette question. Cela a même été la première démarche que nous avons entreprise. Nous avons également, dès le début, demandé des explications à l'ambassadeur des Etats-Unis en France, ainsi qu'une audience, là encore sans aucun résultat. Chacun sait qu'aux termes de négociations dont le contenu, pour l'instant, reste en partie opaque, il a fallu presque trois ans à compter de l'arrestation des Français par les forces américaines en Afghanistan et au Pakistan, pour que quatre 
d'entre eux, fin juillet 2004, soient remis par les autorités américaines aux autorités françaises.

6 Au-delà, on ne peut que relever le décalage entre d'une part la capacité de mobilisation et/ou d'indignation dont savent faire preuve certains politiques français et quelques belles consciences devant certains évènements, et d'autre part leur silence assourdissant s'agissant de la situation des Français de Guantanamo.

7 C\&C - Est-ce que vous pensez que le fait de mobiliser l'opinion publique pourrait être un moyen de faire changer l'attitude des pouvoirs publics français concernant la question des prisonniers à Guantanamo ?

8 Oui, peut-être. A priori, tout ce qui pouvait susciter un intérêt pour ce dossier ne pouvait que nous aider à sortir de la léthargie que nous avons connue jusqu'à l'été 2004. Nous avions, à un moment donné, envisagé de lancer une grande pétition internationale à partir de la France. Mais d'une part les autorités américaines auraient été totalement indifférentes à ce type d'initiative et d'autre part, le spectre du terrorisme international et les effets pervers qu'il génère, rendent compliquée une mobilisation en France sur le sort de jeunes musulmans dans un contexte dans lequel une partie de l'opinion publique française considère que les mauvais traitements qu'ils ont subis à Guantanamo, si condamnable soient-ils, s'éclipsent dès lors qu'ils sont désignés, sans preuve aucune, comme ayant pu vouloir embrasser la cause du terrorisme international. Cela ne nous a pas découragés de prendre d'autres initiatives. En effet, en octobre 2003, nous avons reçu un avis du Rapporteur Spécial de l'ONU sur la détention que nous avions saisi. Celui-ci a considéré que la situation de Guantanamo devait être considérée comme une forme de détention arbitraire. Mais au vu du mépris des autorités américaines pour les institutions onusiennes, cela ne nous aidera probablement pas beaucoup. Nous avons également saisi le Rapporteur Spécial sur la torture et les mauvais traitements de la même sous-commission et restons dans l'attente du premier résultat de ses travaux. La presse internationale a divulgué, courant décembre 2004, des extraits d'un rapport du CICR stigmatisant le fait que les traitements subis à Guantanamo par les détenus devaient être considérés, pour certains d'entre eux, comme caractéristiques de tortures et de mauvais traitements. Les avocats anglais et américains qui ont interviewé les détenus, notamment anglais, qui avaient été libérés ont conclu exactement dans le même sens que le CICR. Mais, pour partie, nous restons encore dans l'ignorance des mauvais traitements qui ont été infligés. Nous avions également, il y a un an, demandé au ministre des Affaires Etrangères, que la France saisisse la Cour Internationale de Justice de la Haye (CIJ) à propos de la violation par les Etats-Unis de différentes conventions internationales et au premier chef les conventions de Genève ainsi que les conventions de Vienne relatives aux visites consulaires $^{3}$. On nous a tout simplement indiqué que pour différentes raisons, la France ne souhaitait pas s'engager dans cette voie. Même si quatre Français ont été rapatriés vers la France à la fin du mois de juillet 2004, nous ne pouvons que constater l'impuissance de la France à obtenir des informations sur les catégories dans lesquelles restent classés trois autres détenus français à Guantanamo (catégorie 1 : cour spéciale, probable peine de mort; catégorie 2 : on soumet le prisonnier concerné au droit commun militaire; catégorie 3 : on le rend à son pays d'origine). Pourtant de telles informations seraient cruciales puisqu'elles permettraient de trancher la question de savoir si certains des prisonniers français sont en risque d'être traduits devant les autorités judiciaires américaines ou non et le cas échéant sont en risque d'être 
condamnés à mort. Il n'y a aucune information sur le sort judiciaire des Français qui restent détenus par les Etats-Unis. Ceci étant, on commence à savoir ce qui s'est passé ne serait-ce qu'en fonction de ce qu'ont raconté à Maître Jacques Debray et à moimême, Messieurs Mourad Benchellali et Nizar Sassi. Leur récit est effrayant. S'il n'y a pas eu d'atteinte grave à leur intégrité physique, l'humiliation qu'ils ont subie, le confinement dans lequel ils ont été maintenus, ne manqueront pas, malheureusement, d'être à l'origine de séquelles psychologiques lourdes. Ils nous ont indiqué avoir été témoins de scènes très choquantes, à savoir les interrogatoires de détenus provenant $\mathrm{du}$ Proche Orient, menés par des jeunes femmes à demi vêtues ou conduits simultanément avec des projections de films pornographiques. Ceci étant, on est obligé de se demander si les prisonniers français n'ont pas été et ne sont pas encore, les victimes collatérales de la volonté de l'administration américaine de punir la France pour son refus de participer à la guerre contre l'Irak. Evidemment, les pouvoirs publics français ne peuvent que le nier. Mais dans l'état actuel des choses, c'est difficile de ne pas s'interroger. Il est vrai que dans le cas de la Grande-Bretagne c'est bien l'opinion publique qui, à la suite de l'évocation de la peine de mort par injection létale à l'encontre de certains prisonniers britanniques, a poussé le gouvernement britannique à exiger des garanties des Etats-Unis. En un sens, ce sursaut de la société civile a bien été un succès relatif puisque la non-application de la peine de mort a été garantie pour ces prisonniers, ainsi que le principe de l'incarcération éventuelle de ces prisonniers en Grande-Bretagne. Mais, il faut aussi voir dans quelles conditions. En effet, cette garantie n'a été appliquée qu'à condition que les prisonniers britanniques en question plaident coupable. Ce type de marchandage est pour le moins scandaleux.

C\&C - Dans ce cadre, est-ce qu'il y a des démarches en faveur d'une coordination entre avocats à l'échelle transnationale?

Il y a eu des rencontres entre avocats européens à Londres et à Bruxelles et nous nous sommes rendus à Washington en janvier 2004, avec Maître Jacques Debray pour rencontrer les grandes organisations de défense des droits de l'Homme américaines $\left(\mathrm{ACLU}, \mathrm{CCR}^{4}\right)$ et des représentants de certains Sénateurs américains. Ceci étant, les cultures et traditions juridiques sont différentes et il est par conséquent très difficile de mettre en place une initiative commune. Ce qui est caractéristique de la France c'est que c'est pour le moment le pays où il $\mathrm{y}$ a eu le plus de procédures. Ces procédures, nous les avons menées avec Me Jacques Debray en essayant dans un premier temps d'amener le juge français à reconnaître sa compétence pour juger de la légalité de la détention des prisonniers français à Guantanamo dans un contexte dans lequel les Etats-Unis refusent d'appliquer les Convention de Genève. Il faut en effet rappeler que la France, ayant ratifié les conventions de Genève, est non seulement tenue de les respecter mais aussi les faire respecter ${ }^{5}$. On a donc considéré que le tribunal de Paris devait se déclarer compétent en la matière et faire injonction à l'administration française de faire respecter les Conventions de Genève. En effet, le juge civil en France (conformément à l'article 66 de la Constitution) est le gardien des libertés. Toute une jurisprudence de la Cour de Cassation le confirme. Nous avons introduit un référé en ce sens devant M. le Président du Tribunal de grande Instance de Paris. Pour des raisons procédurales compliquées cela n'a pas marché. Le juge des référés s'est déclaré incompétent. C'est vrai qu'il n'y avait aucun précédent. Cependant, nous avons tenté la même chose dans une configuration juridique différente devant le tribunal de Lyon. Nous avons finalement reporté cette procédure compte tenu de l'annonce à l'été 2004 du transfert de certains prisonniers français à Guantanamo. En effet, il ne semblait pas 
judicieux de poursuivre sur cette voie dans le contexte du transfert de quatre des détenus français à Guantanamo. Cependant une bataille juridique a été engagée devant la Cour de cassation et son issue est décisive puisqu'il s'agit de la seule procédure pénale engagée contre l'administration américaine à l'heure actuelle. En effet, en automne 2002, nous avons déposé une plainte pour détention illégale auprès de $\mathrm{M}$. le Doyen des juges d'instruction du Tribunal de Lyon ${ }^{6}$. En réponse, le juge d'instruction de Lyon a prononcé en février 2003 une ordonnance de refus d'informer au motif que les agents d'Etat américains, comme tout fonctionnaire public, ne peuvent faire l'objet d'une poursuite devant une juridiction pénale d'un Etat étranger. Cette décision proclame donc une immunité générale des agents publics et des responsables de l'exécutif d'un Etat étranger? ${ }^{7}$. Nous avons fait appel de cette décision et nous avons joint à cet appel un avis rendu par deux professeurs de droit international, M. Alain Pellet, qui représente la France à la Cour Internationale de Justice, et M. Hervé Ascensio. Mais le 20 mai 2003, la Cour d'appel de Lyon a confirmé le refus d'information en ajoutant à la motivation du juge d'instruction un autre argument juridique qui mérite quelques commentaires. Ce dernier consistait en effet à dire que les résolutions 1368 et 1373 du Conseil de Sécurité de l'ONU du 12 et 28 septembre 2001 respectivement, faisant obligation aux Etats de lutter contre le terrorisme, mais aussi l'art. 51 de la charte de l'ONU (droit à la légitime défense des Etats), ne permettaient pas de dire que la détention des Français à Guantanamo est arbitraire. Eu égard à ces résolutions et à la charte, ces détentions ne pouvaient selon la Cour d'appel être considérées comme arbitraires. On est là au cœur du problème qui est la manière dont l'argument de la lutte contre le terrorisme permet d'éroder l'Etat de droit et de priver les juges de leur rôle de garant des libertés. Il faut bien voir que ces deux décisions (celle du juge d'instruction et de la Cour d'appel), rendent le droit humanitaire de la guerre, notamment concernant les prisonniers de guerre, parfaitement inapplicable. La motivation de la Cour d'appel de Lyon va même plus loin puisqu'elle consiste à dire que, même à supposer que les détentions à Guantanamo soient illégales ou illicites, leur illégalité ou leur illéicité disparaît devant l'obligation des Etats de lutter contre le terrorisme. L'argument est d'une immense gravité. De plus l'argument relatif aux résolutions du Conseil de Sécurité est fallacieux puisque ceux-ci ne sont pas directement applicables en droit français. Enfin, si l'on retient l'hypothèse du juge d'instruction français sur l'immunité générale de tous les fonctionnaires publics, les procès contre les agents de la Stasi ou les agents libyens impliqués dans l'affaire Lockerbie auraient été illégaux. C'est absurde. D'un point de vue juridique, ces décisions sont donc discutables ${ }^{8}$. En réalité, on a refusé nos requêtes pour des raisons politiques évidentes. La justice française est malheureusement parfois traversée par une logique de raison d'Etat et ce par la pression qu'exerce parfois la hiérarchie sur les magistrats. C'était vrai il y a trente ans et c'est peut-être encore vrai aujourd'hui... Ainsi, cette décision incroyable a été soumise à la Cour de Cassation et la bataille juridique est engagée. Il faut espérer que la Cour de Cassation aura le courage de casser la décision de la Cour d'appel de Lyon. Elle doit rendre son arrêt le 4 janvier 20059. Si elle le faisait, comme d'ailleurs Monsieur l'Avocat Général l'a invitée à le faire, cela pourrait aboutir à ce qu'un juge d'instruction Lyon se saisisse des faits de séquestration et détention arbitraire subis par les Français à Guantanamo.

11 C\&C - On a l'impression que l'administration américaine a mis en place un état d'exception en jouant sur d'un côté le droit international et la notion de prisonnier de guerre, de l'autre côté le droit interne et la notion de criminel. 
Autrefois, on était soit l'un, soit l'autre. Maintenant on est ni l'un, ni l'autre, et les prisonniers se retrouvent finalement entre ces deux catégories...

Cette question de l'absence de statut juridique appliqué à ces prisonniers est au centre de la bataille menée par les avocats américains dans la Cour suprême et dans les différentes juridictions fédérales. Jusqu'à une date récente, ils avaient échoué mais une grande victoire essentielle a été obtenue quand la Cour Suprême des Etats-Unis le 28 juin 2004 a décidé de poser des limites au pouvoir de guerre du Président Georges Bush. Dorénavant, les prisonniers considérés comme des terroristes, ont le droit de saisir la justice américaine, ce qu'ils ont commencé à faire avec l'aide de nombreux avocats américains. Jusqu'à cette date, il y avait eu un certain nombre de décisions de justice validant les choix des « Docteur Folamour » du droit que sont les conseillers juridiques de Rumsfeld. La difficulté d'appliquer le droit interne américain tient à ce que ces conseillers n'ont bien évidemment pas choisi la base de Guantanamo par hasard. Elle a été choisie parce que c'est une zone de non-droit. Ainsi, un certain nombre de juges américains ont considéré qu'à Guantanamo la loi américaine (ainsi que toutes les garanties constitutionnelles qu'elle offre) ne trouvait pas application puisqu'il ne s'agit pas d'un territoire sous souveraineté américaine. La même raison a été invoquée quand les autorités américaines ont dit aux autorités françaises que la Conventions de Vienne sur les relations consulaires, qui prévoit des visites consulaires pour les étrangers arrêtés sur un territoire donné, ne trouvaient pas application ${ }^{10}$. En effet, là encore l'argument juridique consistait à dire qu'il ne s'agissait pas d'un territoire américain. Dans ce contexte, les grandes associations de défense des droits de l'homme ont engagé, avec des premiers succès, une bataille constitutionnelle, notamment avec le CCR. L'espoir est qu'une fois passée la paralysie post-traumatique du 11 septembre, les juges américains cesseront eux-mêmes d'être tétanisés et par conséquent reprendront le contrôle de leur mission, ce qu'ils ont commencé à faire avec l'arrêt rendu par la Cour Suprême le 28 juin 2004 qui censure l'attitude de l'administration américaine.

C\&C - On nous dit que la coopération franco-américaine marche très bien entre services de renseignement. Au contraire, la coopération judiciaire est quasiinexistante. Il faut donc bien se poser des questions sur le rôle des juges. Alors que certains juges britanniques semblent très préoccupés par la situation des prisonniers à Guantanamo, les juges français de la section anti-terroriste ne semblent pas mécontents de ce qu'il y ait des prisonniers français à Guantanamo. Qu'en pensez-vous?

Je ne crois pas qu'on puisse dire qu'ils soient mécontents de la situation actuelle. Une information confiée au Juge antiterroriste visant deux des prisonniers français a été ouverte à Paris, sous le chef d'inculpation d'« association de malfaiteur en relation avec une entreprise terroriste » en novembre 2002. Dans ce cadre, la convention judiciaire franco-américaine aurait pu permettre aux Juges d'Instruction d'entendre certains des Français détenus à Guantanamo. Pourtant, ils n'ont jamais pris une telle initiative et n'ont, pas plus, délivré de mandat d'arrêt international. En d'autres termes, il ne semble pas que les juges antiterroristes français aient été particulièrement intéressés par l'idée d'utiliser leur marge de manœuvre judiciaire pour promouvoir ou même préserver les droits de l'Homme. Pour ce qui est de la coopération judiciaire de manière générale, tout ce que je peux dire c'est qu'il y a beaucoup de méfiance du côté des autorités judiciaires américaines vis à vis de la France. Mais, alors que les conventions de Genève stipulent que les prisonniers de guerre doivent être relâchés à la fin de la 
guerre, la " guerre contre le terrorisme » n'a pas de fin en vue aux dires de MM. Bush et Rumsfeld. S'agissant du transfert de Guantanamo vers la France de quatre des détenus français, cela résulte notamment du fait que l'Administration Américaine a tiré les conséquences du renversement de tendance judiciaire qu'a impliqué l'arrêt rendu par la Cour Suprême des Etats-Unis le 28 juin 2004, sans préjudice évidemment des résultats des initiatives prises par les pouvoirs publics français. Ceci étant, des conditions ont dû être mises à la libération des quatre premiers détenus français de Guantanamo. En effet, Georges Bush a été ulcéré par le fait que la Grande-Bretagne ait relâché les prisonniers britanniques après leur transfert. Il faut bien dire qu'aucune charge n'avait pu être retenue contre eux. Dans le cas, des prisonniers français, il y a eu probablement un marché entre l'administration française et le gouvernement américain pour qu'en contrepartie du transfert, les prisonniers français soient écroués en France, gage peut-être de la libération annoncée mais toujours pas effective des trois autres Français (Ridouane Khalid, Khaled Ben Mustapha et Moustak Ali Patel - NDLR) qui restent détenus à Guantanamo pour des raisons absolument obscures. Restent également obscures les conditions dans lesquelles les services de renseignement français et par conséquent des officiers de police judiciaire, qui faisaient partie des délégations françaises qui ont visité les détenus français à Guantanamo, ont procédé à leur interrogatoire, hors le cadre fixé par le Code de procédure pénale, interrogatoires en quelques sortes clandestins, qui ont «nourri » la procédure française dans des conditions qui vont nous amener, avec Maître Jacques Debray, à en contester la régularité.

15 Au-delà, la grande difficulté de ce dossier reste que, par l'addition d'un certain nombre de sophismes et de raisonnements qui procèdent plus de l'amalgame que d'une interprétation objective des faits, les jeunes Français de Vénissieux sont, à notre sens, très hâtivement désignés comme des " apprentis terroristes " par le seul fait de s'être rendus en Afghanistan. Bien d'autres grilles de lecture sont possibles, qui s'appuient notamment sur des ressorts psychologiques et sociologiques dans un contexte qui n'est pas exempt de manipulations. Cela aujourd'hui en France est quasiment indicible et inaudible. Il faudra un jour faire un bilan de l'action des juges antiterroristes en France. Chacun se souvient que dans le procès Chalabi, il y a quatre ans, plus de la moitié des prévenus a été relaxée, certains après avoir subi des années de détention provisoire. On le voit bien, la lutte contre le terrorisme effrite ici ou là un certain nombre de principes fondamentaux du procès équitable et au premier chef la présomption d'innocence ; ce n'est pas le moindre des enjeux de la situation des Français détenus à Guantanamo.

\section{NOTES}

1. Art. 5 de la Convention (III) de Genève relative au traitement des prisonniers de guerre du 12 août 1949 : « s'il y a un doute sur l'appartenance à l'une des catégories énumérées à l'article 4 des personnes qui ont commis un acte de belligérance et qui sont tombées aux mains de l'ennemi, lesdites personnes bénéficieront de la protection de la présente convention en attendant que leur statut ait été déterminé par un tribunal compétent ». 
2. En effet, l'article. 4 de la Convention (IV) de Genève affirme: «Sont protégées par la Convention les personnes qui, à un moment quelconque et de quelque manière que ce soit, se trouvent, en cas de conflit ou d'occupation, au pouvoir d'une Partie au conflit ou d'une Puissance occupante dont elles ne sont pas ressortissantes ».

3. Voir la note 11, NDLR.

4. Respectivement American Civil Liberties Union et Center for Constitutional Rights.

5. Art.1. commun aux quatre conventions de Genève relatives au traitement des prisonniers de guerre: « Les Hautes Parties contractantes s'engagent à respecter et à faire respecter la présente Convention en toutes circonstances".

6. Cette plainte a été déposée sur le fondement de l'art. 432-4 du Code Pénal (une personne dépositaire de l'autorité publique ou chargée d'une mission de service public, agissant dans l'exercice de ses fonctions, et qui a ordonné ou accompli arbitrairement un acte attentatoire à la liberté individuelle est passible de 7 années d'emprisonnement correctionnel) et de l'art 432-5 du Code Pénal ( vise le fait pour un agent public qui a eu connaissance d'une privation de liberté dont l'illégalité est alléguée de s'abstenir d'y mettre un terme alors qu'il avait les moyens et le mandat de la faire). Cette plainte visait donc les administrations françaises afin de les inciter à prendre les dispositions nécessaires pour mettre un terme à l'emprisonnement illégal des prisonniers français à Guantanamo.

7. Cette décision a toutefois précisé que la France peut poursuivre des fonctionnaires publics pour crime de génocide, crime contre l'humanité et crime de guerre, y compris un chef d'Etat en exercice, dans le cadre de sa collaboration avec la Cour Pénale Internationale.

8. Cela est d'autant plus vrai qu'un arrêt de la CIJ du 14 février 2002 ("Arrêt Yerodia »), a priori défavorable à une compétence universelle, contredit pourtant le principe de l'immunité générale des agents publics: cet arrêt interdit à la Belgique de poursuivre l'exécution d'un mandat d'arrêt international qu'elle avait fait délivrer contre un ministre des Affaires Etrangères de Laurent Kabila pour crime contre l'humanité. L'arrêt affirme en effet qu'au vu du droit coutumier international les chefs d'Etat et les ministres des Affaires Etrangères bénéficient d'une immunité. Mais en même temps il contredit ainsi le principe de l'immunité générale des agents publics.

9. Le 4 janvier 2005, La Cour de Cassation cassera l'arrêt rendu par la chambre de l'instruction de la Cour d'appel de Lyon et ordonnera l'ouverture d'une enquête pénale sur la détention de français à Guantanamo soupçonnée d'être illégale en droit français comme dans le droit international.

10. Selon cette Convention conclue à Vienne en 1963 : «Les fonctionnaires consulaires ont le droit de se rendre auprès d'un ressortissant de l'Etat d'envoi qui est incarcéré, en état de détention préventive ou toute autre forme de détention, de s'entretenir et de correspondre avec lui et de pourvoir à sa représentation en justice. Ils ont également le droit de se rendre auprès d'un ressortissant de l'Etat d'envoi qui, dans leur circonscription, est incarcéré ou détenu en exécution d'un jugement » (art. 36.1).

\section{RÉSUMÉS}

Dans cet entretien, Me William Bourdon, avocat de deux des Français ayant été détenus dans le camp de Guantanamo et désormais, depuis leur transfert en France en juillet 2004, mis en examen et écroués pour " association de malfaiteurs en relation avec une entreprise terroriste ", s'exprime sur les évènements marquants et les décisions judiciaires qui ont marqué le « dossier 
Guantanamo » aussi bien en France qu'au niveau international. Il y retrace de manière détaillée les enjeux juridiques et politiques de la détention arbitraire dans le camp américain, en montrant comment elle s'est nourrie d'une suspension de certaines des dispositions fondamentales du droit international ainsi que d'un certain nombre de principes constitutifs du droit au procès équitable. Il y met ainsi en évidence l'érosion du principe de l'indépendance des juges qu'a entraîné le recours à l'argument de la lutte contre le terrorisme après le 11 septembre, tout en ne négligeant pas de souligner que des marges de manœuvre demeurent toutefois à la résistance des juges contre ces pratiques d'exception.

William Bourdon is the lawyer of the two French men detained at Gantanamo and who are since their transfer to France in July 2004 detained for «terrorist activities ». In this interview he refers to the outstanding events and the judicial decisions that have had an impact on the Guantanamo file, in France and internationally. He details the judicial and political stakes on the arbitrary detention in the American camp by showing how it was fed by the suspension of some fundamental dispositions of International Law as well as a certain number of constitutive principles of the right to a fair trial. He thereby shows the erosion of the principle of the independence of the judges engendered by the use of the argument of fight against terrorism since September $11^{\text {th }}$. Yet he does not omit to emphasize that the judges still can resist to these practices of exception.

\section{INDEX}

Mots-clés : camp, détention arbitraire, Entretien, Guantanamo, lutte anti-terroriste, sécurité intérieure

\section{AUTEURS}

\section{DIDIER BIGO}

Didier BIGO est Maître de Conférences des Universités à l'IEP de Paris. Chercheur associé au Centre d'Etudes et de Recherches Internationales (CERI) et Président du Centre d'Etudes sur les Conflits, il dirige les programmes cadres de recherche et développement européens ELISE (European Liberty and Security, PCRD5) et CHALLENGE (Changing the Landscape of European Security, PCRD6).

\section{CHRISTIAN OLSSON}

Christian OLSSON est doctorant en sciences politiques (relations internationales) à l'institut d'Etudes politiques de Paris rattaché au CERI. Il travaille notamment sur les enjeux de reconstruction politique de sociétés (post-)conflictuelles dans les interventions militaires contemporaines (Kosovo, Afghanistan, Iraq)William BOURDON est avocat au Barreau de Paris. En tant qu'ancien secrétaire général de la Fédération Internationale des Droits de l'Homme (FIDH), il est notamment spécialisé dans les questions relatives aux droits de l'Homme. Il est par ailleurs le fondateur de l'association Sherpa, réseau international de juristes constitué pour répondre aux formes d'impunité générées par la mondialisation. Il est l'avocat de deux des Français ayant été détenus dans le camp de Guantanamo. 